\title{
Leitlinienreport zur aktualisierten S2k-Leitlinie der Deutschen \\ Gesellschaft für Gastroenterologie, Verdauungs- \\ und Stoffwechselkrankheiten (DGVS) zu Komplikationen der Leberzirrhose
}

\author{
AWMF-Register Nr. 021-017
}

Autoren

Petra Lynen Jansen ${ }^{1}$, Alexander L. Gerbes ${ }^{2}$, Joachim Labenz ${ }^{3}$, Julia Jungnitsch ${ }^{1}$, Pia van Leeuwen ${ }^{1}$

Institute

1 Deutsche Gesellschaft für Gastroenterologie, Verdauungsund Stoffwechselkrankheiten, Berlin

2 Medizinische Klinik und Poliklinik II, Leber Zentrum des Universitätsklinikums München, München

3 Innere Medizin mit Schwerpunkt Gastroenterologie, Diakonie Klinikum Jung-Stilling, Siegen
Bibliografie

DOI https://doi.org/10.1055/a-0873-4682

Z Gastroenterol 2019; 57: e121-e129

(c) Georg Thieme Verlag KG, Stuttgart · New York

ISSN 0044-2771

Korrespondenzadresse

Petra Lynen Jansen DGVS

Deutsche Gesellschaft für Gastroenterologie, Verdauungs-

und Stoffwechselkrankheiten, Gastro Haus, Olivaer Platz 7,

10707 Berlin

Tel.: ++49/30/3198315003

lynen@dgvs.de

\begin{tabular}{|c|c|c|}
\hline \multicolumn{2}{|c|}{ Inhaltsverzeichnis } & \multirow{2}{*}{$\begin{array}{l}\text { Seite } \\
\text { e121 }\end{array}$} \\
\hline 1. & Geltungsbereich und Zweck & \\
\hline 1.1 & Auswahl des Leitlinienthemas & e121 \\
\hline 1.2 & Zielorientierung der Leitlinie & e121 \\
\hline 1.3 & Patientenzielgruppe & e121 \\
\hline 1.4 & Versorgungsbereich & e122 \\
\hline 1.5 & Anwenderzielgruppe & e122 \\
\hline 2. & $\begin{array}{l}\text { Zusammensetzung der Leitliniengruppe und } \\
\text { Beteiligung von Interessensgruppen }\end{array}$ & e122 \\
\hline 3. & Methodologische Exaktheit & e123 \\
\hline 3.1 & Literaturrecherche und Auswahl der Evidenz & e123 \\
\hline 3.2 & $\begin{array}{l}\text { Formulierung der Empfehlungen und strukturierte } \\
\text { Konsensfindung }\end{array}$ & e123 \\
\hline 3.3 & Zeitplan & e124 \\
\hline 4. & Externe Begutachtung und Verabschiedung & e124 \\
\hline 5. & $\begin{array}{l}\text { Redaktionelle Unabhängigkeit und Umgang mit } \\
\text { potenziellen Interessenkonflikten }\end{array}$ & e124 \\
\hline 6. & Verbreitung und Implementierung & e124 \\
\hline 7. & Gültigkeitsdauer und Aktualisierungsverfahren & e124 \\
\hline \multicolumn{2}{|c|}{$\begin{array}{l}\text { Anhang: Interessenkonflikt-Erklärungen - Tabellarische } \\
\text { Zusammenfassung }\end{array}$} & e125 \\
\hline
\end{tabular}

\section{Geltungsbereich und Zweck}

\subsection{Auswahl des Leitlinienthemas}

Nach Ablauf der Gültigkeit wurde die Leitlinie von 2011 aktualisiert. Da auch die hepatische Enzephalopathie eine schwerwiegende Komplikation der Leberzirrhose darstellt und diese in der alten Leitlinie bisher nicht behandelt wurde, wurde die neue Leitlinie um dieses Kapitel ergänzt.

\subsection{Zielorientierung der Leitlinie}

Die Lebensqualität und die Lebenserwartung von Patienten mit Leberzirrhose ist besonders durch die daraus resultierenden Komplikationen begrenzt. Ziel dieser Leitlinienaktualisierung war es, die in der alten Leitlinie ausgesprochene Empfehlungen auf der Basis einer aktuellen Literaturrecherche zu überprüfen, gegebenenfalls zu korrigieren oder durch neue Empfehlungen zu ersetzen und zu ergänzen, um eine optimierte Versorgung dieser Patienten zu ermöglichen

\subsection{Patientenzielgruppe}

Die Leitlinie gibt Empfehlungen für erwachsene Patienten mit Aszites, hepatorenalem Syndrom, spontan bakterieller Peritonitis, hepatischem Hydrothorax und hepatopulmonalem Syndrom sowie hepatischer Enzephalopathie. 


\subsection{Versorgungsbereich}

Die Leitlinie adressiert sowohl den stationären als auch den ambulanten Versorgungsbereich.

\subsection{Anwenderzielgruppe}

Die Leitlinie richtet sich an alle Ärzte, die Patienten mit Komplikationen der Leberzirrhose versorgen oder an der Versorgung dieser Patienten beteiligt sind, insbesondere Hausärzte, Internisten, Gastroenterologen, Infektiologen, Nephrologen, Neurologen, Chirurgen, Pathologen, Radiologen, Ernährungsmediziner.

\section{Zusammensetzung der Leitliniengruppe und Beteiligung von Interessensgruppen}

Die Leitlinie wurde federführend durch die Deutsche Gesellschaft für Gastroenterologie, Verdauungs- und Stoffwechselkrankheiten (DGVS) erstellt, die als Koordinatoren Herrn Professor Gerbes, München, und Herrn Professor Labenz, Siegen, beauftragte. Methodisch verantwortlich waren Frau PD Dr. med. Lynen Jansen, Frau Dr. Julia Jungnitsch und Frau Pia van Leeuwen, DGVSGeschäftsstelle, Berlin. Frau Dr. Nothacker und Frau Dr. Blödt (beide Arbeitsgemeinschaft der Wissenschaftlichen Medizinischen Fachgesellschaften e. V. [AWMF], Berlin) standen zur methodischen Beratung zur Seite und moderierten als neutrale Leitlinienexpertinnen das Kickoff-Treffen und die Konsensuskonferenz. Eric Wohlfarth, Torsten Karge und Paul Freudenberger standen für das Leitlinienportal zur Verfügung.

Das Leitlinienvorhaben wurde in der Zeitschrift für Gastroenterologie ausgeschrieben und auf der Webseite der AWMF veröffentlicht, sodass weitere Fachgesellschaften/Vertreter sich zur Mitarbeit melden konnten. Die für das Fachgebiet relevanten Fachgesellschaften und Patientengruppen wurden angeschrieben und um die Nennung von Mandatsträgern gebeten. Folgende Fachgesellschaften/Interessengruppen nahmen neben der DGVS stimmberechtigt an der Leitlinienarbeit teil:

- Deutsche Gesellschaft für Allgemein- und Viszeralchirurgie (DGAV) Jens Werner, Andreas Schnitzbauer

- Deutsche Gesellschaft für Innere Medizin e. V. (DGIM) Joachim Labenz

- Deutsche Gesellschaft für Infektiologie e. V. (DGI) Bernd Salzberger, Christoph Lübbert

- Deutsche Gesellschaft für Pathologie e. V./Bundesverband deutscher Pathologen e. V. (DGP/BDP)

Beate Straub, Andrea Tannapfel

- Deutsche Röntgengesellschaft e. V. (DRG)

Andreas Schreyer, Guido Kukuk

- Deutsche Gesellschaft für Interventionelle Radiologie und minimal-invasive Therapie (DeGIR)

Bernhard Meyer

- Deutsche Gesellschaft für Nephrologie (DGFN) Alexander R. Rosenkranz

- Deutsche Gesellschaft für Ultraschall in der Medizin (DEGUM) Andrej Potthoff, André Ignee
- Deutsche Gesellschaft für Neurologie e. V. (DGN) Manuel Dafotakis

- Deutsche Gesellschaft für Ernährungsmedizin e. V. (DGEM) Mathias Plauth

- Lebertransplantierte Deutschland e. V. Jutta Riemer, Egbert Trowe

Die Deutsche Gesellschaft für Pneumologie und Beatmungsmedizin e. V. (DGP) wurde eingeladen, nahm aber nicht an der Leitlinienarbeit teil.

Bei der personellen Besetzung der einzelnen Arbeitsgruppen wurden, wenn möglich, Fachkompetenz, eine interdisziplinäre Verteilung und der jeweilige Tätigkeitsbereich (niedergelassen und stationär) berücksichtigt. Die Leitlinie richtet sich an Internisten, Gastroenterologen, Infektiologen, Nephrologen, Neurologen, Chirurgen, Pathologen, Radiologen und Ernährungsmediziner und dient zur Information für Hausärzte.

\section{Folgende AG-Einteilung wurde beschlossen:}

\section{AG 1: Diagnostik des Aszites}

- AG-Leiter

- Beate Appenrodt: DGVS

- AG-Mitglieder

- Peter Buggisch: DGVS

- André Ignee: DEGUM

- Bernhard Meyer: DeGIR

- Beate K. Straub: DGP/BDP

\section{AG 2: Therapie des Aszites}

- AG-Leiter

- Christian Johannes Steib: DGVS

- Jonel Trebicka: DGVS

- AG-Mitglieder

- Matthias J. Bahr: DGVS

- Bernhard Meyer: DeGIR

- Mathias Plauth: DGEM

- Andrej Potthoff: DEGUM

- Martin Rössle: DGVS

- Jens Werner: DGAV

AG 3: Spontan bakterielle Peritonitis

- AG-Leiter

- Reiner Wiest: DGVS

- Alexander Zipprich: DGVS

- AG-Mitglieder

- Tony Bruns: DGVS

- Christoph Lübbert: DGI

- Bernd Salzberger: DGI

AG 4: Hepatorenales Syndrom

- AG-Leiter

- Veit Gülberg: DGVS

- AG-Mitglieder

- Guido Gerken: DGVS

- Alexander R. Rosenkranz: DGFN

- Marcus-Alexander Wörns: DGVS 
AG 5: Hepatischer Hydrothorax und hepatopulmonales Syndrom

- AG-Leiter

- Matthias Dollinger: DGVS

- AG-Mitglieder

- André Ignee: DEGUM

- Andreas Anton Schnitzbauer: DGAV

- Andreas G. Schreyer: DRG

- Andrea Tannapfel: DGP/BDP

AG 6: Diagnostik der Hepatischen Enzephalopathie

- AG-Leiter

- Felix Gundling: DGVS

- Gerald Kircheis: DGVS

- AG-Mitglieder

- Manuel Dafotakis: DGN

- Guido Kukuk: DRG

- Marcus Schuchmann: DGVS

- Matthias Wettstein: DGVS

AG 7: Therapie der Hepatischen Enzephalopathie

- AG-Leiter

- Axel Holstege: DGVS

- AG-Mitglieder

- Manuel Dafotakis: DGN

- Manfred Gross: DGVS

- Guido Kukuk: DRG

- Johann Ockenga: DGVS

- Mathias Plauth: DGEM

- Michael Schepke: DGVS

\section{Methodologische Exaktheit}

\subsection{Literaturrecherche und Auswahl der Evidenz}

Basis dieser Aktualisierung war die Leitlinie von 2011. Auf einem ersten Treffen (Kickoff-Treffen) der Koordinatoren, Mandatsträger und der Arbeitsgruppenleiter wurden die Inhalte und das methodische Vorgehen festgelegt.

Durch die Koordinatoren wurde vorab ein Katalog von Schlüsselfragen erarbeitet, die in der Leitlinie beantwortet werden sollten. Die Schlüsselfragen wurden sieben Themengebieten/Arbeitsgruppen zugeordnet, diskutiert, ergänzt und verabschiedet. Im Vorfeld des ersten Treffens führten die Koordinatoren außerdem eine Suche nach verfügbaren Quellen aggregierter Evidenz durch. Existierende Leitlinien und Metaanalysen wurden auf dem Kickoff-Treffen vorgestellt. Die erweiterte Evidenzrecherche erfolgte in der Pubmed und der Cochrane-Datenbank durch die AG-Leiter. Weitere Artikel und Studien konnten bei Bedarf mit einbezogen werden. Literatur wurde bis zum Termin der Konsensuskonferenz berücksichtigt.

\subsection{Formulierung der Empfehlungen und strukturierte Konsensfindung}

Auf Grundlage der Literatur wurden die Empfehlungen und Hintergrundtexte durch die AG-Leiter erarbeitet und zunächst im E-Mail-Umlaufverfahren innerhalb der einzelnen AGs abgestimmt.
- Tab. 1 Schema zur Graduierung von Empfehlungen. Negative Empfehlungen werden entsprechend formuliert.

\begin{tabular}{|l|l|}
\hline Syntax & Beschreibung \\
\hline soll & starke Empfehlung \\
\hline sollte & Empfehlung \\
\hline kann & Empfehlung offen \\
\hline
\end{tabular}

- Tab. 2 Konsensfindung.

\begin{tabular}{|l|c|}
\hline Konsens & \% Zustimmung \\
\hline starker Konsens & $>95$ \\
\hline Konsens & $>75-95$ \\
\hline mehrheitliche Zustimmung & $50-75$ \\
\hline kein Konsens & $<50$ \\
\hline
\end{tabular}

Die Graduierung der Empfehlungen erfolgte über die Formulierung soll, sollte, kann ( $\triangleright$ Tab. 1$)$.

Alle Empfehlungen wurden dann zunächst in einem Delphi-Verfahren von allen Leitlinienmitarbeitern mithilfe einer 5-stufigen Entscheidungsskala abgestimmt (ja, eher ja, unentschieden, eher nein, nein). Zu Empfehlungen, die nicht mit ja/eher ja abgestimmt wurden, musste ein begründender Kommentar hinterlegt werden. Empfehlungen, die zu über $95 \%$ mit ja/eher ja abgestimmt wurden, konnten bereits zu diesem Zeitpunkt verabschiedet werden ( $\vee$ Tab. 2).

Die Kommentare und Änderungsvorschläge der Delphi-Runde wurden von den AG- Leitern und den Koordinatoren gesichtet und die Empfehlungen überarbeitet. Die Empfehlungen der AG 6 und 7 wurden komplett neu erarbeitet und abgestimmt. In einer strukturierten, zweitägigen Konsensuskonferenz unter unabhängiger Moderation von Frau Nothacker und Frau Blödt (AWMF) stellten die AG-Leiter die überarbeiteten Empfehlungen vor. Diese wurden nach den Prinzipien der NIH-Konferenz besprochen und abgestimmt: Präsentation im Gesamtplenum unter Berücksichtigung der Kommentare und ggf. Erläuterungen durch die AGLeiter, Aufnahme von Stellungnahmen und ggf. Änderung, Abstimmung, Festschreiben des Ergebnisses.

Diskutiert wurden:

- alle Empfehlungen, die in der Delphi-Runde weniger als 95\% Zustimmung erhalten hatten

- Empfehlungen, die inhaltlich verändert wurden

- Empfehlungen, die bereits in der Delphi-Runde verabschiedet worden waren, aber aufgrund von Dopplungen oder zur Verbesserung der inhaltlichen Stringenz der Leitlinie in den Kommentar verschoben wurden

- neue Empfehlungen

Empfehlungen, die in der Delphi-Runde nicht verabschiedet und in den Kommentarteil verschoben wurden, wurden nicht erneut abgestimmt. Ebenfalls nicht abgestimmt wurden Empfehlungen, die unverändert aus der Leitlinie von 2011 übernommen werden konnten. 
Enthaltungen zu den einzelnen Abstimmungen wurden dokumentiert. Die Konsensusstärke wurde gemäß > Tab. 2 festgelegt. Im Anschluss an die Konsensuskonferenz erfolgte die finale Überarbeitung der Kommentare durch die AG-Leiter und die redaktionelle Zusammenstellung der Leitlinie durch die Koordinatoren.

\subsection{Zeitplan}

\begin{tabular}{|l|l|}
\hline April 2016 & $\begin{array}{l}\text { Ausschreibung in der ZFG und Anmeldung bei } \\
\text { der AWMF }\end{array}$ \\
\hline Dezember 2016 & Beauftragung der Koordinatoren durch die DGVS \\
\hline Februar 2017 & Kickoff-Treffen Berlin \\
\hline September 2017 & Delphi-Verfahren \\
\hline Januar 2018 & Konsensuskonferenz Berlin \\
\hline
\end{tabular}

\section{Externe Begutachtung und Verabschiedung}

Die Leitlinie wurde allen beteiligten Fachgesellschaften zur Stellungnahme vorgelegt und von diesen verabschiedet. Durch die AWMF erfolgte eine externe formale Beurteilung.

\section{Redaktionelle Unabhängigkeit und Um- gang mit potenziellen Interessenkonflikten}

Die Leitlinie wurde ausschließlich von der DGVS finanziert. Vertreter der pharmazeutischen Industrie wurden nicht am Prozess der Leitlinienentwicklung beteiligt, um Neutralität und Unabhängigkeit zu wahren. Mandatsträger und Experten arbeiteten ausschließlich ehrenamtlich.

Vor Beginn der Konsensuskonferenz legten alle Teilnehmer ihre Interessen offen (Anhang). Hierfür wurden Interessenkonflikte schriftlich mithilfe eines Formblattes der AWMF, das materielle direkte und indirekte Interessen umfasst, erfasst und der Leitliniengruppe tabellarisch zur Verfügung gestellt. Die Interessenkonflikte wurden im Vorfeld von Herrn Gerbes, Frau Nothacker und Frau Lynen gesichtet und nach den Kategorien geringfügig, moderat und schwer bewertet. Interessenkonflikte mit Bezug zu der Leitlinie, die

- Berater- bzw. Gutachtertätigkeit oder bezahlte Mitarbeit in einem wissenschaftlichen Beirat eines Unternehmens der Gesundheitswirtschaft (z. B. Arzneimittelindustrie, Medizinproduktindustrie), eines kommerziell orientierten Auftragsinstituts oder einer Versicherung

- finanzielle Zuwendungen (Drittmittel) für Forschungsvorhaben oder direkte Finanzierung von Mitarbeitern der Einrichtung vonseiten eines Unternehmens der Gesundheitswirtschaft, eines kommerziell orientierten Auftragsinstituts oder einer Versicherung

- Eigentümerinteresse an Arzneimitteln/Medizinprodukten (z. B. Patent, Urheberrecht, Verkaufslizenz)

- Besitz von Geschäftsanteilen, Aktien, Fonds mit Beteiligung von Unternehmen der Gesundheitswirtschaft

betreffen, wurden als moderate Konflikte eingestuft.
Teilnehmer der Konsensuskonferenz waren Beate Appenrodt, Matthias J. Bahr, Susanne Blödt, Tony Bruns, Peter Buggisch, Matthias Dollinger, Paul Freudenberger, Alexander L. Gerbes, Manfred Gross, Veit Gülberg, Felix Gundling, Axel Holstege, André Ignee, Julia Jugnitsch, Torsten Karge, Gerald Kircheis, Guido Kukuk, Joachim Labenz, Christoph Lübbert, Petra Lynen, Bernhard Meyer, Monika Nothacker, Matthias Plauth, Alexander R. Rosenkranz, Bernd Salzberger, Michael Schepke, Marcus Schuchmann, Christian J. Steib, Beate K. Straub, Jonel Trebicka, Egbert Trowe, Matthias Wettstein, Reiner Wiest, Marcus-Alexander Wörns, Alexander Zipprich.

Im Einzelnen wiesen folgende Personen moderate Konflikte auf:

\begin{tabular}{|l|l|}
\hline Alexander L. Gerbes & Alexander R. Rosenkranz \\
\hline Manfred Gross & Marcus Schuchmann \\
\hline Felix Gundling & Christian Johannes Steib \\
\hline Gerald Kircheis & Beate K. Straub \\
\hline Joachim Labenz & Jonel Trebicka \\
\hline Christoph Lübbert & Marcus-Alexander Wörns \\
\hline & Bernhard Meyer \\
\hline
\end{tabular}

Anton Schnitzbauer, Marcel Dafotakis und Johann Ockenga wurden ebenfalls als Personen mit moderaten Konflikten eingestuft, waren aber auf der Konsensuskonferenz entschuldigt nicht anwesend.

Die Leitliniengruppe entschied einstimmig, dass die von dieser Einschätzung betroffenen Personen sich bei Abstimmungen über Empfehlungen, die von diesen Interessenkonflikten direkt berührt werden könnten, enthalten.

Honorare für Vortrags- und Schulungstätigkeiten oder bezahlte Autoren- oder Co-Autorenschaften im Auftrag eines Unternehmens der Gesundheitswirtschaft, eines kommerziell orientierten Auftragsinstituts oder einer Versicherung wurden als geringfügig eingestuft und führten nicht zur Notwendigkeit einer Enthaltung.

\section{Verbreitung und Implementierung}

Die Leitlinie sowie der Leitlinienreport werden auf der Homepage der DGVS (www.dgvs.de) und der AWMF (www.awmf.org) zum freien Download zur Verfügung gestellt. Die Langversion der Leitlinie wird in der „Zeitschrift für Gastroenterologie“ in deutscher Sprache publiziert. Die Leitlinienempfehlungen werden darüber hinaus auf den Kongressen und themenbezogenen Fortbildungsveranstaltungen der DGVS vorgestellt. Eine englischsprachige Version der Leitlinie ist geplant.

\section{Gültigkeitsdauer und Aktualisierungs- verfahren}

Die Gültigkeit der Leitlinie beträgt fünf Jahre (2023). Eine Überarbeitung der Leitlinie bei veränderter Datenlage erfolgt gegebenenfalls auch früher. Das Aktualisierungsverfahren wird koordiniert durch die DGVS-Geschäftsstelle. 


\section{Anhang: Interessenkonflikt-Erklärungen - Tabellarische Zusammenfassung}

1. Berater- bzw. Gutachtertätigkeit oder bezahlte Mitarbeit in einem wissenschaftlichen Beirat eines Unternehmens der Gesundheitswirtschaft (z. B. Arzneimittelindustrie, Medizinproduktindustrie), eines kommerziell orientierten Auftragsinstituts oder einer Versicherung

2. Honorare für Vortrags- und Schulungstätigkeiten oder bezahlte Autoren- oder Co- Autorenschaften im Auftrag eines Unternehmens der Gesundheitswirtschaft, eines kommerziell orientierten Auftragsinstituts oder einer Versicherung

3. finanzielle Zuwendungen (Drittmittel) für Forschungsvorhaben oder direkte Finanzierung von Mitarbeitern der Einrichtung vonseiten eines Unternehmens der Gesundheitswirtschaft, eines kommerziell orientierten Auftragsinstituts oder einer Versicherung
4. Eigentümerinteresse an Arzneimitteln/Medizinprodukten (z. B. Patent, Urheber- recht, Verkaufslizenz)

5. Besitz von Geschäftsanteilen, Aktien, Fonds mit Beteiligung von Unternehmen der Gesundheitswirtschaft

6. persönliche Beziehungen zu einem Vertretungsberechtigten eines Unternehmens der Gesundheitswirtschaft

7. Mitglied von in Zusammenhang mit der Leitlinienentwicklung relevanten Fachgesellschaften/Berufsverbänden, Mandatsträger im Rahmen der Leitlinienentwicklung

8. politische, akademische (z. B. Zugehörigkeit zu bestimmten „Schulen“), wissenschaftliche oder persönliche Interessen, die mögliche Konflikte begründen könnten

9. gegenwärtiger Arbeitgeber, relevante frühere Arbeitgeber der letzten 3 Jahre

\begin{tabular}{|c|c|c|c|c|}
\hline & Appenrodt, Beate & Bahr, Matthias J. & Bruns, Tony & Buggisch, Peter \\
\hline 1 & - & - & $\begin{array}{l}\text { ja: Beratertätigkeit Intercept } \\
\text { Pharma Deutschland GmbH }\end{array}$ & ja: Abbvie, Gilead, Janssen, MSD \\
\hline 2 & ja: Firma Falk & $\begin{array}{l}\text { ja: Vortragshonorare } \\
\text { (Falk Foundation) }\end{array}$ & $\begin{array}{l}\text { ja: Referentenhonorare von Falk } \\
\text { und Norgine GmbH }\end{array}$ & $\begin{array}{l}\text { ja: Abbvie, Gilead, Falk, BMS, } \\
\text { Merz, MSD }\end{array}$ \\
\hline 3 & - & - & - & - \\
\hline 4 & - & - & - & - \\
\hline 5 & - & - & - & - \\
\hline 6 & - & - & - & - \\
\hline 7 & & $\begin{array}{l}\text { ja: Mitgliedschaften: Deutsche } \\
\text { Gesellschaft für Verdauungs- und } \\
\text { Stoffwechselkrankheiten (DGVS), } \\
\text { Berufs- verband Gastroenterolo- } \\
\text { gie Deutschland (BVG), Arbeits- } \\
\text { gemeinschaft Leitender Gastroen- } \\
\text { terologischer Krankenhausärzte } \\
\text { (ALGK), Deutsche Arbeitsgemein- } \\
\text { schaft zum Studium der Leber } \\
\text { (GASL), European Association for } \\
\text { the Study of the Liver (EASL), } \\
\text { Norddeutsche Gesellschaft für } \\
\text { Gastroenterologie (NDGG) }\end{array}$ & - & ja: BNG, DGVS \\
\hline 8 & - & - & - & - \\
\hline \multirow[t]{2}{*}{9} & Universitätsklinik des Saarlandes & Sana Kliniken Lübeck GmbH & Universitätsklinikum Jena & selbstständig \\
\hline & Dafotakis, Manuel & Dollinger, Matthias & Gerbes, Alexander L. & Gerken, Guido \\
\hline 1 & $\begin{array}{l}\text { ja: Beratertätigkeit (advisory } \\
\text { boards) für Pfizer und Merz. }\end{array}$ & ja: Gilead, AbbVie, Nordiag, Bayer & ja: Berater CSL Behring & $\begin{array}{l}\text { ja: MSD, BMS, Gilead, Falk, } \\
\text { Humedics, Bayer, Abbvie }\end{array}$ \\
\hline 2 & $\begin{array}{l}\text { ja: Vorträge für Pfizer, Merz, Al- } \\
\text { lergan, Ipsen, Böhringer, Daiichi- } \\
\text { Sankyo. }\end{array}$ & ja: Falk, Gilead, AbbVie & $\begin{array}{l}\text { ja: Falk Foundation, CSL Behring, } \\
\text { Gilead }\end{array}$ & $\begin{array}{l}\text { ja: MSD, BMS, Gilead, Falk, } \\
\text { Humedics, Bayer, Abbvie }\end{array}$ \\
\hline 3 & - & ja: Merck, Perspectum & - & $\begin{array}{l}\text { ja: BMS, BTG, Humedics, Boston } \\
\text { Sci }\end{array}$ \\
\hline 4 & - & - & - & - \\
\hline 5 & - & - & - & - \\
\hline 6 & - & - & - & - \\
\hline 7 & - & ja: DGVS & ja: DGVS-Mitglied & ja: DGVS \\
\hline 8 & - & - & - & - \\
\hline 9 & Uniklinik Aachen & $\begin{array}{l}\text { Klinikum Landshut gGmbH, } \\
\text { Universitätsklinikum Ulm }\end{array}$ & Klinikum der Universität München & Universitätsklinikum Essen \\
\hline
\end{tabular}




\begin{tabular}{|c|c|c|c|c|}
\hline & Gross, Manfred & Gülberg, Veit & Gundling, Felix & Holstege, Axel \\
\hline 1 & $\begin{array}{l}\text { ja: Advisory Board für Norgine } \\
\text { GmbH }\end{array}$ & - & - & - \\
\hline 2 & $\begin{array}{l}\text { ja: Vorträge für die Falkfounda- } \\
\text { tion, für Medical Tribune, für } \\
\text { Omniamed, für Norgine GmbH, } \\
\text { für Reckitt Benckiser, Pfizer, BMS }\end{array}$ & - & $\begin{array}{l}\text { ja: Honorare für Vortragstätigkeit } \\
\text { bei Fortbildungsveranstaltungen } \\
\text { sowie Advisory Boards von } \\
\text { Norgine, Merz, Abbvie }\end{array}$ & - \\
\hline 3 & $\begin{array}{l}\text { ja: Teilnahme an Studien von } \\
\text { Novartis }\end{array}$ & - & $\begin{array}{l}\text { ja: DFG gesponserte Studie } \\
\text { (WET-Studie, Weight Loss } \\
\text { Endoscopy Trial) }\end{array}$ & - \\
\hline 4 & - & - & - & - \\
\hline 5 & - & - & - & ja: GILEAD \\
\hline 6 & - & - & - & - \\
\hline 7 & $\begin{array}{l}\text { ja: Mitglied DGIM, DEGUM, aber } \\
\text { kein Mandatsträger }\end{array}$ & - & ja: DGVS, DDG, BDI & ja: DGVS \\
\hline 8 & - & - & - & - \\
\hline \multirow[t]{2}{*}{9} & $\begin{array}{l}\text { Internistisches Klinikum } \\
\text { München Süd }\end{array}$ & Klinikum der Universität, München & $\begin{array}{l}\text { Klinikum Bogenhausen, Städt- } \\
\text { isches Klinikum München GmbH }\end{array}$ & $\begin{array}{l}\text { Klinikum Landshut (bis 6/2016) } \\
\text { seitdem berentet }\end{array}$ \\
\hline & Ignee, André & Kircheis, Gerald & Kukuk, Guido & Labenz, Joachim \\
\hline 1 & - & $\begin{array}{l}\text { ja: Zum einen war ich in den Jahren } \\
\text { von } 1991 \text { bis } 1999 \text { wissenschaftli- } \\
\text { cher Mitarbeiter der Firma MERZ \& } \\
\text { Co. In dieser Zeit habe ich die } \\
\text { meisten der publizierten Daten } \\
\text { zum L- Ornithin-L-Asparat erarbei- } \\
\text { tet. In den nachfolgenden Jahren } \\
\text { habe ich sowohl Vorträge zum L- } \\
\text { Ornithin-L- Asparat auf nationalen } \\
\text { und internationalen Kongressen, } \\
\text { aber auch auf Einladung der Firma } \\
\text { gehalten, welche durch meine de- } \\
\text { taillierte Kenntnis auf genanntem } \\
\text { Gebiet bedingt war. Zum zweiten } \\
\text { bin ich mit drei anderen Wissen- } \\
\text { schaftlern Patentinhaber für ein } \\
\text { Gerät zur Messung der Flimmer- } \\
\text { frequenz. Dieses Gerät wurde } \\
\text { durch eine Studie zur Validierung } \\
\text { der Flimmerfrequenz im Rahmen } \\
\text { der Hepatischen Enzephalopathie } \\
\text { entwickelt (tragbare Variante der } \\
\text { Schuhfriedschen Flimmerfrequenz } \\
\text { Messvorrichtung). }\end{array}$ & - & $\begin{array}{l}\text { ja: Norgine, Advisory Board } \\
\text { national und international Andere } \\
\text { hier nicht relevant, da kein Bezug } \\
\text { zu Lebererkrankungen }\end{array}$ \\
\hline 2 & $\begin{array}{l}\text { ja: Referate für die Firma Boston, } \\
\text { Supersonic, HItachi, Bracco }\end{array}$ & $\begin{array}{l}\text { ja: Schulungstätigkeit für den } \\
\text { HEPAtonorm Analyzer (Funk- } \\
\text { tionsweise, Messung der Flim- } \\
\text { merfrequenz). Vortragstätigkeit } \\
\text { zur Therapie der HE }\end{array}$ & $\begin{array}{l}\text { ja: Bayer Healthcare, Leverkusen, } \\
\text { Deutschland: gelegentliche } \\
\text { bezahlte Vorträge und Work- } \\
\text { shops (max. 2000,- Euro/Jahr) } \\
\text { Philips Healthcare, Best, Nieder- } \\
\text { lande: gelegentliche bezahlte } \\
\text { Vorträge und Workshops (max. } \\
\text { 2000,- Euro/Jahr) }\end{array}$ & $\begin{array}{l}\text { ja: Norgine, Vortragshonorare, } \\
\text { Publikationshonorar Merz, } \\
\text { Publikationshonorar }\end{array}$ \\
\hline 3 & - & - & - & - \\
\hline 4 & - & ja: siehe Punkt 2 & - & - \\
\hline 5 & - & - & - & - \\
\hline 6 & - & - & - & - \\
\hline 7 & - & - & - & $\begin{array}{l}\text { ja: Mitglied DGVS, DGIM, BVGD, } \\
\text { BDI, ALGK, Mandat der DGIM }\end{array}$ \\
\hline 8 & - & - & - & - \\
\hline
\end{tabular}




\begin{tabular}{|c|c|c|c|c|}
\hline 9 & $\begin{array}{l}\text { Caritas-Krankenhaus Bad } \\
\text { Mergentheim }\end{array}$ & $\begin{array}{l}\text { 1. Heinrich-Heine- Universität } \\
\text { Düsseldorf, } 40225 \text { Düsseldorf, } \\
\text { Moorenstraße 5; 2. Städtisches } \\
\text { Klinikum Brandenburg GmbH, } \\
\text { Hochschulklinikum der MHB } \\
\text { „Theodor Fontane“, Hochstraße } \\
29,14770 \text { Brandenburg an der } \\
\text { Havel }\end{array}$ & Universitätsklinikum Bonn & $\begin{array}{l}\text { Diakonie Klinikum, Jung-Stilling- } \\
\text { Krankenhaus Siegen }\end{array}$ \\
\hline & Lübbert, Christoph & Lynen Jansen, Petra & Meyer, Bernhard & Nothacker, Monika \\
\hline 1 & ja: MSD Sharp \& Dohme & - & - & $\begin{array}{l}\text { ja: IQTIG Beratung; IQWIG } \\
\text { Beratung Arzneimittel ATINO6 }\end{array}$ \\
\hline 2 & - & - & $\begin{array}{l}\text { ja: Vortragstätigkeiten für die } \\
\text { Fa. W. L. Gore, Bayer AG, Siemens } \\
\text { Healthcare GmbH, Merit Medical }\end{array}$ & $\begin{array}{l}\text { ja: BÄK Nordrhein QM + LL, } \\
\text { Cochrane-Manual }\end{array}$ \\
\hline 3 & ja: Astellas, Da Volterra, Shionogi & - & $\begin{array}{l}\text { ja: Forschungskooperation } \\
\text { (Auftragsforschung für Siemens } \\
\text { Healthcare } \mathrm{GmbH} \text { ) }\end{array}$ & $\begin{array}{l}\text { ja: BÄK Expertise Leitlinien + } \\
\text { Individ. }\end{array}$ \\
\hline 4 & - & - & - & - \\
\hline 5 & - & - & - & - \\
\hline 6 & - & - & - & - \\
\hline 7 & ja: DGVS, DGIM, DGI, PEG, DTG & - & $\begin{array}{l}\text { ja: Mitglied der Deutschen } \\
\text { Röntgengesellschaft (DRG), der } \\
\text { Deutschen Gesellschaft für Inter- } \\
\text { ventionelle Radiologie (DEGIR) }\end{array}$ & $\begin{array}{l}\text { ja: Sprecherin des FB LL des } \\
\text { DNEbM }\end{array}$ \\
\hline 8 & - & - & - & ja: evidenzbasierte Medizin \\
\hline 9 & Universitätsklinikum Leipzig & DGVS, RWTH Aachen & $\begin{array}{l}\text { Medizinische Hochschule } \\
\text { Hannover, Land Niedersachsen }\end{array}$ & AWMF \\
\hline & Ockenga, Johann & Plauth, Mathias & Potthoff, Andrej & Riemer, Jutta \\
\hline 1 & $\begin{array}{l}\text { ja: Fresenius Kabi GmbH, Advisory } \\
\text { Board zum Studien- design einer } \\
\text { parenteralen Ernährung bei } \\
\text { onkologischen Patienten }\end{array}$ & - & - & - \\
\hline 2 & $\begin{array}{l}\text { ja: Honorare für Vorträge von: } \\
\text { Abbvie, Abbott, Bayer, Baxter, } \\
\text { Braun, Bristol Myers Squibb, Frese- } \\
\text { nius, GHD, Nutricia, Roche, GILEAD }\end{array}$ & $\begin{array}{l}\text { ja: Honorare für ärztliche Fortbil- } \\
\text { dungen: AbbVie, B Braun Melsun- } \\
\text { gen, Falk Foundation e. V., Frese- } \\
\text { nius Kabi Deutschland, Shire }\end{array}$ & $\begin{array}{l}\text { ja: Referententätigkeit: Toshiba } \\
\text { Medical Systems, Neuss, Germany }\end{array}$ & - \\
\hline 3 & - & $\begin{array}{l}\text { ja: Fresenius Kabi Deutschland: } \\
\text { Forschungsprojekt Krankenhaus- } \\
\text { mangelernährung }\end{array}$ & - & - \\
\hline 4 & - & - & - & - \\
\hline 5 & - & - & - & - \\
\hline 6 & - & - & - & - \\
\hline 7 & $\begin{array}{l}\text { ja: Mitglied der DGVS, DGEM, } \\
\text { Präsident der DGEM, Beirat } \\
\text { Gastro-Liga }\end{array}$ & $\begin{array}{l}\text { ja: Deutsche Gesellschaft für } \\
\text { Ernährungsmedizin e. V. (DGEM), } \\
\text { Mandatsträger }\end{array}$ & - & - \\
\hline 8 & - & - & - & - \\
\hline \multirow[t]{2}{*}{9} & $\begin{array}{l}\text { Gesundheit Nord GmbH, Klini- } \\
\text { kum Bremen Mitte }\end{array}$ & Städtisches Klinikum Dessau & $\begin{array}{l}\text { Medizinische Hochschule Hanno- } \\
\text { ver Klinik für Gastroenterologie, } \\
\text { Hepatologie und Endokrinologie, } \\
\text { Carl- Neuberg-Str. 1, } 30625 \text { Han- } \\
\text { nover, Deutschland }\end{array}$ & $\begin{array}{l}\text { Lebertransplantierte Deutschland } \\
\text { e. V. }\end{array}$ \\
\hline & Rosenkranz, Alexander & Rössle, Martin & Salzberger, Bernd & Schepke, Michael \\
\hline 1 & ja: FWF, DGF & $\begin{array}{l}\text { ja: Gutachtertätigkeit Fa. Bentley, } \\
\text { Hechingen, für die Entwicklung } \\
\text { eines beschichteten balloon- } \\
\text { expandierbaren Stents. }\end{array}$ & $\begin{array}{l}\text { ja: Honorare für Beratungstätig- } \\
\text { keit für die Unternehmen GSK, } \\
\text { Sanofi und Roche }\end{array}$ & - \\
\hline
\end{tabular}




\begin{tabular}{|c|c|c|c|c|}
\hline 2 & $\begin{array}{l}\text { ja: Astellas, Astra- Zeneca, } \\
\text { Baxter/Gambro, Bristol- Myers } \\
\text { Squibb, Boehringer, Chiesi, } \\
\text { Fresenius Medical Care, GSK, } \\
\text { Medice, Mitsubishi, MSD, } \\
\text { Novartis, Otsuka, Sandoz }\end{array}$ & - & $\begin{array}{l}\text { ja: Honorare für die Schulungstä- } \\
\text { tigkeiten bzw. Vorträge von } \\
\text { AbbVie, Chiesi, Jansen }\end{array}$ & $\begin{array}{l}\text { ja: HepatoUpdate, jährlich seit } \\
2015\end{array}$ \\
\hline 3 & ja: Fresenius, Baxter & - & $\begin{array}{l}\text { ja: Forschungsprojekte im } \\
\text { Auftrag von GSK, Biochryst }\end{array}$ & - \\
\hline 4 & - & - & - & - \\
\hline 5 & - & - & - & - \\
\hline 6 & - & - & - & - \\
\hline 7 & - & - & $\begin{array}{l}\text { ja: DGI, Mandat für die Leitlinien- } \\
\text { entwicklung der DGI, ESCMID, } \\
\text { DGIM (ohne Mandat) }\end{array}$ & ja: Mitglied DGVS, RWGIM \\
\hline 8 & - & - & - & - \\
\hline \multirow[t]{2}{*}{9} & Medizinische Universität Graz & $\begin{array}{l}\text { Selbstständig, niedergelassener } \\
\text { Arzt. Konsiliartätigkeit an der } \\
\text { Uniklinik Freiburg und dem Städt. } \\
\text { Klinikum, Saarbrücken. }\end{array}$ & Universitätsklinikum Regensburg & Helios Klinikum Siegburg \\
\hline & Schnitzbauer, Andreas Anton & Schreyer, Andreas & Schuchmann, Marcus & Steib, Christian \\
\hline 1 & $\begin{array}{l}\text { ja: Novartis Advisory Board TEVA } \\
\text { Advisory Board Sequanamedical } \\
\text { Advisory Board }\end{array}$ & - & ja: Advisory Board: Norgine & $\begin{array}{l}\text { ja: Advisory Board AbbVie Round } \\
\text { Table Diskussion Gilead }\end{array}$ \\
\hline 2 & ja: Astellas Vortrag & $\begin{array}{l}\text { ja: Vorträge und Workshops für } \\
\text { Bayer Healthcare Takeda }\end{array}$ & $\begin{array}{l}\text { ja: Vortragshonorare - Gilead, } \\
\text { Abbvie, Falk, Norgine }\end{array}$ & $\begin{array}{l}\text { ja: Vortragstätigkeit Firma Abb- } \\
\text { Vie Vortragstätigkeit Firma Gilead } \\
\text { Vortragstätigkeit Firma Gore }\end{array}$ \\
\hline 3 & - & - & - & $\begin{array}{l}\text { ja: Liver Research Fellowship } \\
\text { (Falk/CSL Behring) Unrestricted } \\
\text { Grant Firma Norgine }\end{array}$ \\
\hline 4 & - & - & - & - \\
\hline 5 & - & - & - & ja: Aktien der Firma Morphosys \\
\hline 6 & - & - & - & - \\
\hline 7 & ja: DGAV, DTG & - & ja: DGVS EASL AASLD ALGK & - \\
\hline 8 & - & - & - & - \\
\hline \multirow[t]{2}{*}{9} & $\begin{array}{l}\text { Universitätsklinikum Frankfurt, } \\
\text { Goethe- Universität Frankfurt am } \\
\text { Main }\end{array}$ & Universitätsklinikum Regensburg & $\begin{array}{l}\text { Gesundheitsverbund Landkreis } \\
\text { Konstanz - Klinikum Konstanz }\end{array}$ & Klinikum der LMU, München \\
\hline & Straub, Beate K. & Tannapfel, Andrea & Trebicka, Jonel & Trowe, Egbert \\
\hline 1 & $\begin{array}{l}\text { ja: } 2016 \text { Beratertätigkeit für } \\
\text { Alexion Pharma GmbH }\end{array}$ & - & $\begin{array}{l}\text { ja: Alexion, WLGore\&Associates, } \\
\text { Sequana MEdical }\end{array}$ & - \\
\hline 2 & $\begin{array}{l}\text { ja: } 2017 \text { von Intercept für Vorträ- } \\
\text { ge zum Thema chronischer } \\
\text { Lebererkrankungen ( } 2 \text { Veranstal- } \\
\text { tungen, Honorar jeweils }\end{array}$ & $\begin{array}{l}\text { ja: Referentenhonorar Pharma- } \\
\text { firmen }\end{array}$ & $\begin{array}{l}\text { ja: Vorträge für Alexion, WLGo- } \\
\text { re\&Associates, Sequana Medical, } \\
\text { BMS, Gilad, Grifols }\end{array}$ & - \\
\hline 3 & - & ja: Drittmittel der DGUV & $\begin{array}{l}\text { ja: Falk Pharma WLGore \& } \\
\text { Associates }\end{array}$ & - \\
\hline 4 & - & - & - & - \\
\hline 5 & - & - & - & - \\
\hline 6 & - & - & - & - \\
\hline 7 & $\begin{array}{l}\text { ja: Mitglied bei DGVS, IAP, DGP, } \\
\text { BVP }\end{array}$ & $\begin{array}{l}\text { ja: DGP, DGVS, BV-Pathologen, } \\
\text { DGE-BV }\end{array}$ & - & - \\
\hline 8 & - & - & - & - \\
\hline
\end{tabular}




\begin{tabular}{|c|c|c|c|c|}
\hline 9 & $\begin{array}{l}\text { Institut für Pathologie, Universi- } \\
\text { tätsmedizin Mainz, Johannes } \\
\text { Gutenberg-Universität Mainz } \\
\text { (seit April 2016) Pathologisches } \\
\text { Institut, Uniklinik Heidelberg, } \\
\text { Ruprecht Karls- Universität } \\
\text { Heidelberg (von Februar } 2005 \text { - } \\
\text { März 2016) }\end{array}$ & Georgius Agricola Stiftung Ruhr & Universitätsklinikum Bonn & - \\
\hline & Werner, Jens & Wettstein, Matthias & Wiest, Reiner & Wohlfarth, Erik \\
\hline 1 & - & - & - & - \\
\hline 2 & - & $\begin{array}{l}\text { ja: Pfizer, Abbvie, Gilead, Falk } \\
\text { (Vertrags- honorare) }\end{array}$ & ja: Boston Scientific & - \\
\hline 3 & - & - & - & - \\
\hline 4 & - & - & - & - \\
\hline 5 & - & - & - & - \\
\hline 6 & - & - & - & - \\
\hline 7 & ja: DGVS, DGAV, DGCH & ja: DGVS, DGIM, DEGVM & - & - \\
\hline 8 & - & - & - & - \\
\hline \multirow[t]{2}{*}{9} & $\begin{array}{l}\text { Klinikum der Universität München } \\
\text { Klinikum für Allgemein-, Viszeral- } \\
\text { und Transplantationschirurgie }\end{array}$ & Klinikum Passau & Inselspital, Universität Bern, CH & CGS \\
\hline & Wörns, Marcus- Alexander & Zipprich, Alexander & & \\
\hline 1 & $\begin{array}{l}\text { ja: Bayer HealthCare, Abbvie, } \\
\text { Bristol-Myers Squibb, Celgene, } \\
\text { Norgine }\end{array}$ & - & & \\
\hline 2 & $\begin{array}{l}\text { ja: Bayer HealthCare, Abbvie, } \\
\text { Bristol-Myers Squibb, Gilead, } \\
\text { Celgene, Norgine }\end{array}$ & $\begin{array}{l}\text { ja: Bayer, Falk, Norgine, Abbvie, } \\
\text { Intercept }\end{array}$ & & \\
\hline 3 & - & - & & \\
\hline 4 & - & - & & \\
\hline 5 & - & - & & \\
\hline 6 & - & - & & \\
\hline 7 & ja: DGVS & ja: DGVS, EASL & & \\
\hline 8 & - & - & & \\
\hline 9 & $\begin{array}{l}\text { Universitätsmedizin der Johannes } \\
\text { Gutenberg-Universität Mainz }\end{array}$ & $\begin{array}{l}\text { Martin-Luther- Universität Halle- } \\
\text { Wittenberg, }\end{array}$ & & \\
\hline
\end{tabular}

